\title{
Dai documenti d'archivio la ricostruzione virtuale della Piazzaforte di Pescara
}

\author{
From Archive Documents the Virtual Reconstruction of the Fortress of Pescara
}

\begin{abstract}
Pasquale Tunzi
Dipartimento di Architettura, Università degli Studi dell'Adriatico “G. d'Annunzio” Chieti-Pescara, Pescara, Italy, tunzi@unich.it
\end{abstract}

\begin{abstract}
When the Unity of Italy occurred, the ancient fortress of Pescara, marked by the river with the same name, was demolished to allow the expansion of the Adriatic city. On the mighty sixteenth century building, eastern defense of the Kingdom of Naples, remain only the eighteenth and nineteenth century maps made by the military. A rigorous study conducted by us on the historic center of Pescara has allowed us to carry out extensive archival surveys and punctual inspections. Then different documents emerged and testimonies that encouraged a virtual reconstruction of the disappeared artefact in which the military garrison and the inhabited nucleus were enclosed. The virtual reconstruction of the fortress that is presented here has been elaborated by resorting the nineteenth century maps, carefully analyzed and compared to perform, critically, a translation of the two-dimensional technical figuration in threedimensional processing. A sort of regeneration of the historical image was thus conducted with the intent of recovering a fundamental element of the city, almost completely forgotten.
\end{abstract}

Keywords: Virtual reconstruction, fortress, Pescara, three-dimensional model.

\section{Introduzione}

Lo studio storico di una città rivela identità nascoste, caratteri perduti, percezioni dimenticate.

Nell'indagare il passato di una città dall'aspetto moderno, come Pescara, sono emerse immagini impensabili e documenti inconsueti riesumati dagli archivi. Proprio questi ultimi, infatti, con la loro preziosissima raccolta di atti e di certificazioni consentono una sempre maggiore definizione della storia, anche di quella attinente a realtà minori. Il valore della documentazione custodita è incommensurabile, essendo un patrimonio culturale diversificato, stratificato e unico che ha scandito il tempo e registrato le azioni degli uomini in determinati luoghi e contesti. La necessità di consultare in modo sistematico tali incartamenti da cui emergono strutture sociali, intenzioni e opere, va perseguita come un dovere sovrastorico, al fine di poter divulgare esempi di vita utili a favorire la crescita culturale della società (Nietzsche, 1874). Si tratta di "memoria" che giova alla definizione di realtà passate, da rievocare, dice Paolo Rossi (1991), in una sorta di scavo antropologico utile all'orientamento di decisioni future.

L'esplorazione anamnestica si rivela necessaria a portare alla luce materiali e situazioni sepolte, in cui notevole contributo è dato dalle immagini. Infatti i disegni, le mappe, le raffigurazioni in genere hanno un potere evocativo e assertivo 
molto forte, nelle quali è insita una via diretta e immediata alla comunicazione.

Per rilevanza storica i documenti d'archivio risultano quindi indispensabili in questa sorta di operazione "archeologica", in quanto sono una risorsa strategica nella conoscenza, soprattutto quando si hanno raffigurazioni di territori trasformati o di edifici perduti, qual è il nostro caso: la Piazzaforte di Pescara. Iniziare dalla distruzione del sistema difensivo di questa città, da ciò che non è più presente, significa inoltrarsi nelle problematiche della storia urbana, attuando il "metodo regressivo" menzionato da Jacques Le Goff (1989), efficace nella ricostruzione del passato urbano partendo dalle tracce attuali.

Il nostro percorso di studio sul centro storico di Pescara è iniziato affrontando il rilievo mensorio dell'esistente, al quale si è presto aggiunta la ricerca d'archivio per una conoscenza più approfondita delle varie unità edilizie. Proprio tale ricerca introdotta interpellando il catasto storico e conseguentemente consultando i documenti depositati presso l'Archivio di Stato di Pescara, ha permesso di ampliare e definire la connotazione del centro storico le cui indagini si sono orientate presso altri archivi.

I primi documenti iconografici da noi rinvenuti sulla Piazzaforte sono presso l'Istituto Storico e di Cultura dell' Arma del Genio (I.S.C.A.G.) di Roma, costituiscono un gruppo di otto mappe realizzate dai militari tra il 1811 e il 1885 , prevalentemente a grande scala e per scopi diversi. Alcune sono l'attestazione di uno stato di fatto, una sorta di perizia eseguita sulla struttura difensiva e sul territorio contermine, altre invece avanzano proposte di progetto per una migliore difesa della costa adriatica e del versante nordorientale del Regno di Napoli. Sono rappresentazioni in cui è contenuto un modo di intendere il rapporto tra territorio, difesa e residenza all'unisono, peraltro significativo del guardare la realtà fisica e di trasferirla su carta secondo principi tecnici propri dell'epoca.

La lettura e l'osservazione delle mappe dell'I.S.C.A.G. ci ha spinti a estendere lo studio recuperando altre mappe coeve conservate altrove. Nell'Archivio di Stato di Napoli e nella Biblioteca Nazionale Vittorio Emanuele III, ad esempio, è custodito un ulteriore corpus di mappe redatto tra Sette e Ottocento, in cui sono registrate le possibili trasformazioni che si sarebbero dovute effettuare, oppure attestano un quadro della struttura difensiva al momento della rilevazione, e in altre mappe ancora vi sono le modifiche effettuate in ottemperanza a precise esigenze.

A queste mappe particolarmente belle si associano i grafici realizzati nei secoli precedenti, sempre per scopi militari, di cui abbiamo testimonianze negli archivi italiani ed europei, a volte riprodotti in pubblicazioni di saggi e monografie. Prendere in considerazione anche queste mappe, seppur in modo sommario, ha reso maggiormente comprensibili le trasformazioni avvenute e i caratteri propri della struttura difensiva di Pescara. Il recupero di tali carte storiche, la lettura e l'analisi, il riordino cronologico dei segni del passato, i sopralluoghi e la consultazione dei trattati militari hanno consentito, in modo dinamico, di affrontare i diversi aspetti del nostro soggetto, e di entrare nei dettagli. Così la traduzione in configurazioni diverse è sorta spontanea, in una visualizzazione maggiormente esaustiva sia dell'insieme che di ogni particolare. Vedere la Piazzaforte in modo alternativo al linguaggio tecnico ha portato alla realizzazione di un modello digitale $3 \mathrm{D}$, successivamente contestualizzato nell'attuale sito urbano a fini divulgativi.

Il patrimonio storico icnografico ha svelato quindi un'altra immagine di Pescara, vissuta per oltre tre secoli e poi cancellata in virtù di un progresso incline a occultare e dimenticare per oltre un secolo il tempo trascorso. Tutto ciò ha favorito una cultura scevra d'identità, tanto da indurre la cittadinanza a pensare di non avere un passato. Pertanto una diffusione appropriata di quell'immagine riesumata dagli archivi oggi si rende necessaria nel tentativo di recuperare un contatto con le proprie radici storiche, ma soprattutto quei valori identitari da condividere.

\section{Le mappe ottocentesche di Pescara}

Quando Carlo V d'Asburgo, intorno alla metà del Cinquecento, volle rinforzare la difesa del Regno di Napoli con una rete di castelli e di torri 
costiere -la cui efficienza fu peraltro citata da Michel de Montaigne nel Journal du VoyagePescara era un piccolo borgo cinto da semplici mura turrite, la cui posizione territoriale le consentì di far parte del piano castellano. Su consiglio del viceré duca d'Alcalà, dopo un'attenta ispezione della costa adriatica effettuata nel 1560 , il nucleo abitato fortificato in età bizantina, per la sua vicinanza al mare e fiancheggiato dal fiume omonimo, fu ritenuto un buon porto fluviale e punto nodale delle linee di traffico commerciale nord-sud e trasversalmente verso il Tirreno e la costa dalmata.

L'abitato di Pescara dovette così assumere il ruolo di Piazzaforte antemurale nord-orientale del Regno, e costituire insieme alla fortezza di Civitella del Tronto e al castello di Ortona un sicuro avamposto. La sua immagine, prima del tutto sconosciuta, iniziò da quel momento a essere riprodotta per soddisfare le esigenze militari e, in modo sempre più attento e tecnico, si andò tracciando una fisionomia dettata dall'occorrenza. Si è via via generato un corpus documentario ampio e interessante, strettamente riservato ai vertici delle forze armate usato come interfaccia grafica, ossia funzionale alla frequentazione e alla gestione della difesa della Piazza e del territorio circostante.

Da uno spedito sguardo alle mappe, suddivise per epoche, si rileva in quelle cinquecentesche una raffigurazione abbastanza schematica e limitata alla fortezza bastionata tagliata dal fiume che scorre in un contesto amorfo, mentre una parte di territorio piuttosto ampia e ben curata nei dettagli caratterizza le mappe della Piazzaforte nel Settecento. Sono evidentemente due modi differenti di osservare e di affrontare la realtà al momento della registrazione grafica che porta con sé una condotta determinata da esigenze specifiche e dalla cultura del momento. Le mappe ottocentesche invece mostrano molto da vicino la fortezza considerandola una macchina bellica che, in alcuni casi, ha relazione con una porzione di territorio, molto circoscritta, essendone la sua diretta estensione. Di queste mappe rinvenute numerose nei diversi archivi italiani ed europei, ci soffermeremo a commentarne alcune per introdurci alla lettura di una di esse in modo specifico.

\subsection{Le mappe dell'I.S.A.G. di Roma}

Prima di passare a osservare con attenzione le mappe romane, è opportuno precisare che ne escluderemo tre non essendo pertinenti al nostro lavoro.

Avviamo la disamina con la "Real Piazza di Pescara" realizzata da Giovanni Melorio ufficiale del Corpo Reale del Genio, divenuto nel 1826 direttore del Reale Officio Topografico di Napoli. Infatti dietro il foglio è scritto a penna: "Officio Topografico dello Stato Magg. Gen. / corrisponde ad altro simile disegno esistenti nell'Officio. 1811" (Fig. 1).

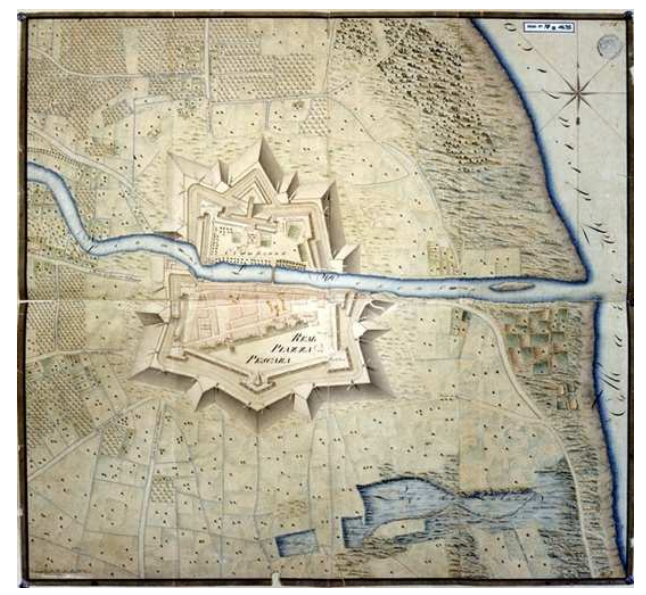

Fig. 1. Real Piazza di Pescara, 1811 (I.S.C.A.G.).

In effetti nella Biblioteca Nazionale di Napoli abbiamo una mappa simile dal titolo "Piano della Piazza di Pescara" realizzata nella prima decade dell'Ottocento. Quest'ultima si differenzia dalla mappa dell'I.S.C.A.G. per la presenza di una legenda esplicativa collegata alle diverse parti del disegno attraverso 38 numeri opportunamente disposti. Quella romana essendo priva di legenda, reca invece i nomi dei sette bastioni e di alcune emergenze direttamente sull'edificato. Entrambe le mappe a colori sono orientate e inquadrano la fortezza con buona parte del territorio circostante sino al mare. Molto probabilmente la nostra mappa $(86 \times 81,5 \mathrm{~cm})$ dotata di scala grafica di 60 tese fu realizzata all'inizio del decennio napoleonico, col proposito di potenziare la Piazza ritenuta di facile presa. Invero, a segui- 
to di una ispezione avviata nel novembre del 1805 dal Capitano d'artiglieria Vincenzo D'Escamard, fu prevista una serie di piccole opere, la cui realizzazione avvenne in parte, come vedremo nella mappa seguente, al fine di esercitare adeguatamente il proprio ruolo.

La fortezza è formata da due aree contrapposte legate da un ponte. Quella a sud occupata dall' agglomerato urbano è protetta sul fiume dalla caserma di fanteria, la cui lunghezza di m 347 collega i bastioni opposti. Circa nel mezzo è attraversata dalla porta principale situata in prossimità del ponte, e si conclude all'estremità orientale con il castello, un piccolo edificio di pianta rettangolare posto di traverso per delimitare gli spazi destinati alle manovre militari. Le cortine bastionate sono libere da protezione avanzate, e soltanto a ovest si rileva la tenaglia spezzata. A nord l'area di Rampinna, destinata alla Cavalleria, reca tra i bastioni dell'opera a corno le tenaglie semplici, e a nord il rivellino avanzato con la retrostante caponiera e falsabraga.

Maggiore ampiezza del campo topografico è nel "Piano della Piazza di Pescara" (115,5 x 70,5 $\mathrm{cm})$ redatto dal tenente di artiglieria Antonio Castellano in data imprecisata (Fig. 2).

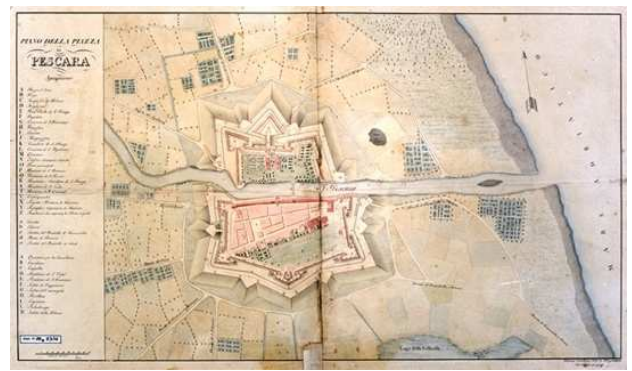

Fig. 2. Piano della Piazza di Pescara (I.S.C.A.G.).

La mappa raffigura, in modo molto simile alla precedente, la fortezza in scala grafica di 100 tese per una migliore lettura delle sue parti, vieppiù sostenuta da una lunga legenda posta sul lato sinistro del foglio. Suddivisa in tre parti, questa permette di rilevare le emergenze e soprattutto le opere militari. A sud tra i bastioni di S. Rocco e di S. Nicola vediamo il cavaliere di S. Thiago sul bastione omonimo già presente nelle mappe precedenti, e alle sue spalle trova luogo l'esteso Prato destinato alle esercitazioni della milizia. Grazie alla legenda è possibile chiarire quanto è presente anche in altre mappe. Tra i bastioni e gli spalti vi sono le cunette continue, all'interno delle quali corre tutt'intorno la fustigata con l'acqua proveniente dal fiume. Ai due estremi ovest ed est della fortezza, attestata sulla riva meridionale del fiume, vi è rispettivamente una lunetta con batteria di fascine e la controguardia a protezione dei rastelli di Chieti e di Francavilla. Accostata alla cortina di occidente, tra il bastione S. Antonio e S. Rocco, sono posti ad antemurale la tenaglia spezzata con caponiera di fascine e sul retro i tamburi utili a coprire le porte segrete nei fianchi ritirati. Inoltre rileviamo l'inserimento di tamburi a ridosso dei fianchi ritirati anche per gli altri bastioni, la cui presenza ci permette di ipotizzare una datazione anteriore al 1809. Particolare evidenza, con un colore più intenso, è dato alle residenze militari e all'ospedale. Infine risultano ben raffigurati il camminamento di ronda con le traverse, le rampe d'accesso ai bastioni e le cannoniere.

Una sorta di ingrandimento della mappa del Melorio è la "Piazza di Pescara", priva di caratterizzazione del territorio, di data, d'autore e scala grafica, fu realizzata a colori su un foglio di carta $(68 \times 63,5 \mathrm{~cm})$ suddiviso in sei parti, di cui due più piccole, incollate su tela. Reca nei due angoli in basso del foglio le legende in formato di tabella dove sono enumerati i fabbricati ad uso militare (ospedale, uffici, magazzini, polveriera, corpi di guardia), con la quantità degli uomini ospitati e i cavalli, e infine il possesso immobiliare che risulta essere demaniale. Si ripetono nei diversi punti della pianta, i nomi dei bastioni e delle principali emergenze. È la prima delle otto mappe in cui si ha soltanto la rappresentazione della fortezza priva del contesto ma attorniata da una serie di strade radiali, più $o$ meno ampie, che la rendono parte di un territorio sfornito di ogni caratterizzazione. Evidentemente l'attenzione è posta sull'intera struttura fortificata e sugli edifici, evidenziati col colore blu quelli destinati alle milizie. La mappa è disegnata con molta cura e non presenta alcuna differenza rispetto alla suddetta in merito alle opere fortificate, pertanto potremmo datarla ai primi tre 
lustri dell'Ottocento e farla rientrare in quel piano di accertamento della difesa finalizzato a un possibile potenziamento.

A seguito di una lunga riflessione da parte della Corona in merito a come tutelare la popolazione dalle frequenti e disastrose inondazioni del fiume, avvenute ancora nel 1839 e due anni dopo (Pessolano, 2006), nel 1849 fu redatta la "Pianta della Piazza di Pescara" (82 x $61 \mathrm{~cm})$. È l'attestazione dello stato della difesa, in cui le sfumature degli spalti mettono in risalto l'articolazione della macchina da guerra rafforzata da tenaglie semplici poste tra i bastoni. La legenda alfabetica sulla destra del foglio precisa i soliti nomi dei bastioni e degli edifici principali, mentre le scale grafiche di 100 canne napoletane e di $200 \mathrm{~m}$ consentono di poter effettuare una prima misurazione dei diversi elementi posti contro uno sfondo piatto.

Molto simile alla suddetta mappa è la "Real Piazza di Pescara", stilata nel 1856 in scala di 100 canne napoletane, anch'essa a colori e disegnata in modo da riempire completamente il foglio $(74 \times 52 \mathrm{~cm})$ suddiviso in otto parti uguali incollate su tela (Fig. 3).

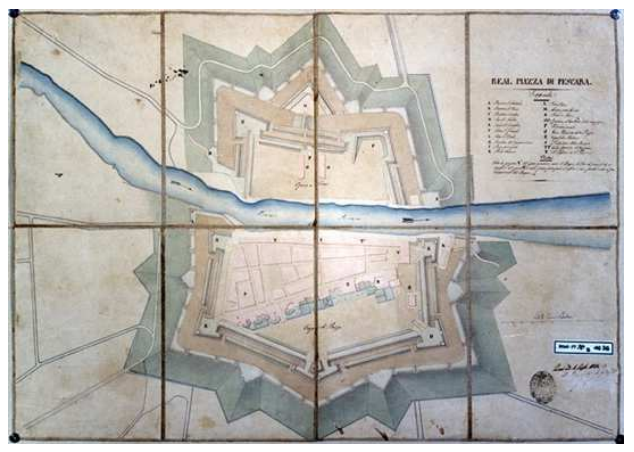

Fig. 3. Real Piazza di Pescara, 1856 (I.S.C.A.G.).

La consueta legenda alfabetica, seppur succinta, consente l'esposizione delle diverse parti edificate. È una raffigurazione chiara della fortezza in cui però sono mancanti molti particolari utili a definire la conformazione puntuale. Tuttavia è precisato che nell'angolo tra castello e caserma vi è l'ingresso al Bagno de' Servi di pena.

Chiudiamo questo excursus citando le successive mappe del 1867 e del 1885, l'una in scala
1:5000, quindi molto piccola nella raffigurazione, e l'altra in cui si documentano le prime demolizioni. Ricordiamo inoltre che a seguito dell'Unità Nazionale il 30 dicembre 1866 fu emanato il decreto di soppressione della Piazzaforte di Pescara.

\section{Lettura dell'icnografia della Piazzaforte}

Come afferma Roberto Potenza (2015) per comprendere i caratteri "della fortezza di Pescara, nei trecento anni di vita che vanno dalla costruzione alla demolizione, è indispensabile un'attenta analisi della cartografia storica ed una continua comparazione con l'attuale conformazione urbana".

Dalle suddette mappe romane e da quanto si è osservato in quelle napoletane emerge quindi l'evidente impossibilità di scegliere una mappa da ricostruire virtualmente in $3 \mathrm{D}$, essendo tutte diverse proprio nei dettagli. Dunque, ne abbiamo prese due, quella di Giovanni Melorio del 1811 e l'ultima disegnata prima dell'Unità Nazionale nel 1856, per affrontare una lettura puntuale e globale delle componenti architettoniche difensive in relazione allo spazio circostante. La prima è una attestazione anteriore alla guerra austriaca, mentre la seconda, essendo prossima alla dismissione funzionale della Piazzaforte, raffigura molto nitidamente tutte le parti della fortificazione, integrandosi alla precedente.

La pianta del 1811, per l'uso del chiaro-scuro, mostra molto bene l'andamento degli spalti che attorniano la fortezza. Questa riporta le numerose cannoniere svasate inserite nei bastioni dai fianchi ritirati e nelle cortine, senza tralasciare le rampe che conducono ai camminamenti, alle piazze dei bastioni protette dai parapetti, agli spalti interrotti dalle traverse. Sul prolungamento di via dei Quartieri la rampa a sinistra conduce alla sommità del bastione sant'Antonio (a ovest) dotato di quattro cannoniere sulle facce, in numero minore rispetto ai più grandi bastioni situati a nord. Analogamente la rampa per accedere al bastione san Rocco (a sud-ovest) era posta sul prolungamento della strada di san Cetteo, l'odierna via dei bastioni. Diversamente disposte erano le due rampe del bastione S. Giacomo (a sud) e san Nicola (a sud-est) situate al centro 
delle rispettive gole, grazie all'ampio spazio consentito dalla piazza d'armi. Le facce di entrambi i bastioni avevano tre cannoniere. La rampa di accesso al bastione san Cristoforo (ad est) era, invece, obliqua perché a poca distanza il castello ne ostacolava il passaggio diretto. Il suo parapetto era forato da quattro cannoniere poste su ognuna delle due facce.

In Rampinna una diversa disposizione avevano le rampe, essendovi maggiore spazio e una diversa disposizione dei bastioni, in particolare quello grande di S. Francesco (a nord-est). Le cannoniere sono in numero di cinque per ogni faccia dei due bastioni. Quest'area a nord del fiume è più contenuta rispetto alla zona sud, difesa dai due bastioni suddetti formanti sul lato settentrionale la tipica coda di rondine, seppur non perfettamente simmetrica, e da un rivellino centrale nel quale si inserisce la caponiera. All'interno dell'area due piccoli edifici di pianta rettangolare destinati alla Cavalleria e una cappella interposta dedicata alla Beata Vergine del Carmelo sono attestati a ridosso della cortina, mentre il resto dello spazio ospita quattro piccoli lotti verdi, una doppia fila di alberi ordinati in margine al fiume e nella cortina occidentale, l'unica Porta denominata de' Cappuccini è costretta nell'angusta ansa del fiume, lì dove la cortina occidentale vi s'innesta.
Inoltre nella mappa del 1856 notiamo tra i bastioni la presenza di tenaglie semplici e sugli spalti un maggior numero di traverse.

\section{Trasmutazione di una icnografía storica in modello 3D}

Per modellare in digitale la Piazzaforte abbiamo separato i diversi elementi contenuti nelle mappe storiche, alla stregua di requisiti. Nella specifica significazione semiologica essi definiscono, come abbiamo visto, il grado della figuratività più o meno accentuato e utile al nostro scopo.

Osservare, confrontare e ridisegnare i diversi elementi della fortezza ci ha portato a effettuare riscontri sui possibili cambiamenti avutisi nel tempo. La lettura puntuale e scrupolosa è stata coadiuvata dalle preziose indicazioni fornite dal Maresciallo Giuseppe Parisi (1802) e dalle incisioni di Giuseppe Guerra all'inizio dell'Ottocento, al fine di poter eseguire una traduzione intersemiotica equilibrata (Fig. 4).

Inoltre una sezione ottocentesca di una porzione della Piazzaforte rinvenuta nell'Archivio di Stato di Pescara, redatta in occasione dei sopralluoghi finalizzati alla demolizione (1873), ha permesso di condurre con maggiore certezza la ricostruzione virtuale in elevato (Fig. 5).

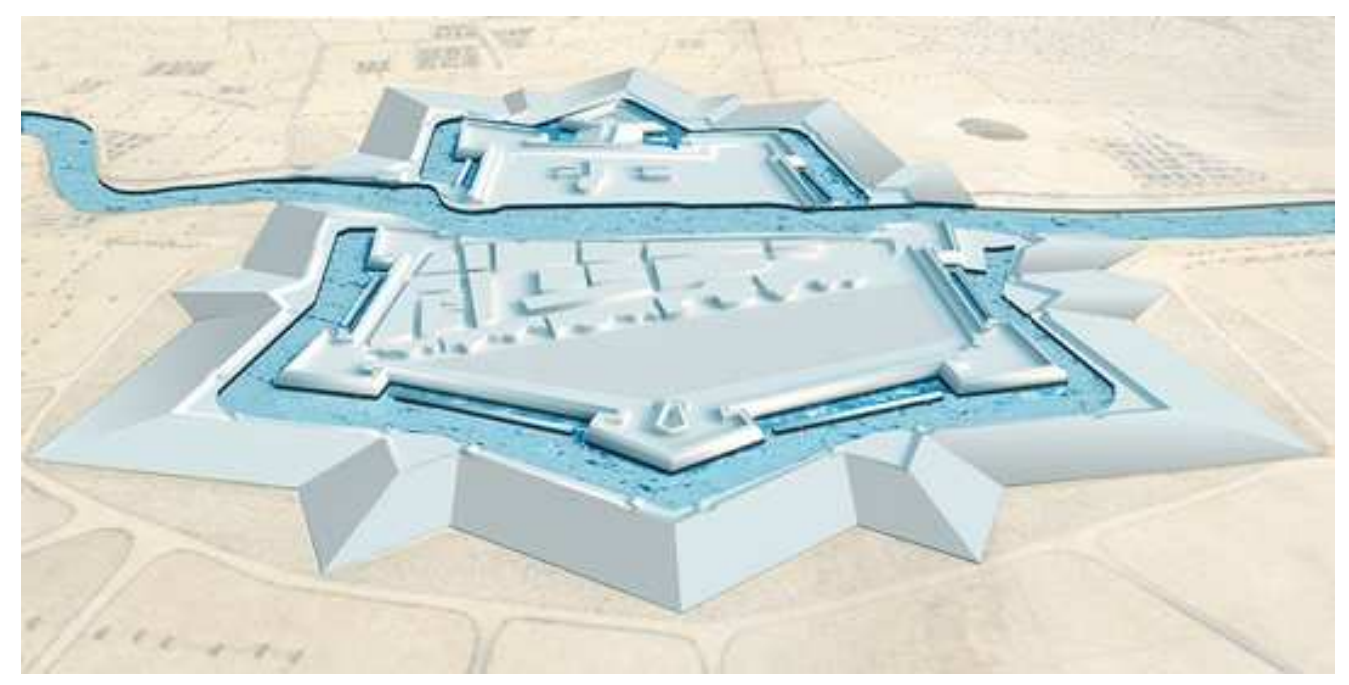

Fig. 4. Elaborazione tridimensionale digitale in itinere della Piazzaforte di Pescara (R. Potenza, P. Tunzi 2014-2018). 


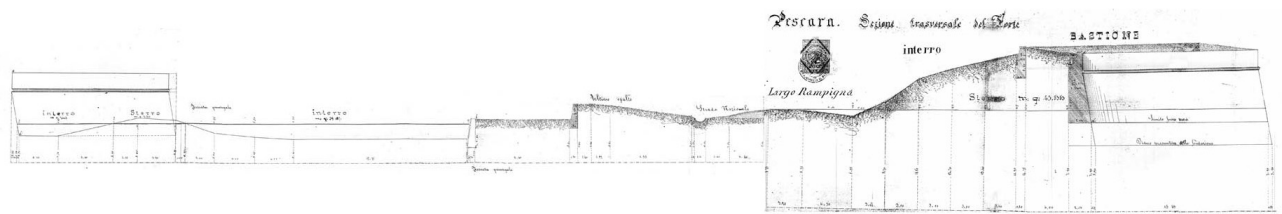

Fig. 5. Pescara, Sezione trasversale del Forte a nord-ovest, 24 giugno 1873 (Archivio di Stato di Pescara).

Siamo stati sempre consapevoli della limitazione imposta dalle mappe che, per quanto belle e affascinanti, non riescono a sollecitare l'immaginazione spaziale, soprattutto in coloro che di architettura militare non ne sanno nulla. Inoltre il linguaggio tecnico della raffigurazione è oggettivamente uno sbarramento alla comprensione dell'immagine. Questo limite andava superato pensando a un'altra figurazione più comunicativa ed eloquente, impiegando le nuove tecniche di raffigurazione digitale con le quali creare immagini esaustive (Giammusso, 2014). Pertanto abbiamo scelto la mappa del 1811 perché vi si ravvisa una certa cura grafica nella raffigurazione di ogni parte più che in altre mappe, e poi, sovrapponendola alla mappa del 1856 ha mostrato la quasi perfetta aderenza dei contorni degli spalti e della piazzaforte interna, con piccole e trascurabili variazioni. Proprio questa buona collimazione ci ha convinti a tenerle insieme e a impiegarle partendo dalla più vecchia per integrare gli elementi aggiunti e modificati nel tempo.

L'elaborazione digitale è stata inoltre supportata da una gran quantità di dati eterogenei analizzati criticamente e verificati di volta in volta, in un continuo passaggio di scala, per poter rintracciare un possibile legame con la città odierna. La mappa catastale del 1886 depositata presso l'Archivio di Stato di Pescara ha permesso dei riscontri sul campo, in quanto raffigura parte delle opere difensive esistenti all'epoca insieme alla nuova espansione urbana.

Questo atto ermeneutico di trasmutazione presuppone un traduttore abile, competente e rigoroso. Del disegno d'epoca non si esegue una mera traduzione visuale attraverso il mezzo digitale, in una forma di simulazione forviante, per quanto affascinante, che traghetti la bidimensionalità verso le tre dimensioni, piuttosto si tratta di un processo in cui si combinano diversi fattori, oggetti, ambiente, funzioni ed esperienze per ottenere un incremento cognitivo del bene stesso. L'operazione di traduzione si muove tra la conoscenza dei caratteri di antiche strutture difensive e la loro visualizzazione in 3D.

Così la produzione di un modello digitale non è una riproduzione di un reale passato, bensì una sua estensione, la cui valenza intersemiotica, viene diffusa per lo più mediante immagini a stampa, oltreché sullo schermo.

Pertanto abbiamo ritenuto opportuno privare il nostro modello di colore e di materiali, elementi visivi irrilevanti non essendo fondamentali per la dimensione piuttosto esigua delle immagini finali a stampa e per la specificità di un soggetto non più esistente (Fig. 6).

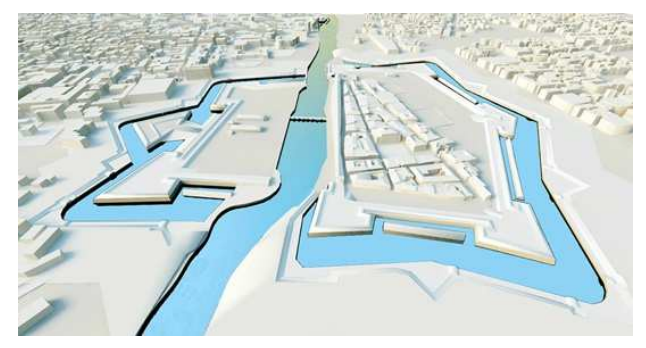

Fig. 6. Modello digitale provvisorio della Piazzaforte di Pescara inserito nel contesto urbano odierno (R. Potenza, P. Tunzi, 2014-2018).

Con tale scelta si è voluto segnare la differenza iconografica nel valore rappresentativo, tra le mappe storiche, oggi riconosciute sul piano artistico, e l'elaborazione computazionale di un soggetto assente fisicamente. Inoltre il modello perde la scala della raffigurazione ma conserva le proporzioni tra le parti, salvando così il valore intrinseco del soggetto che abbiamo voluto relazionare all'attuale sito urbano.

\section{Conclusioni}

Nella redazione di una mappa l'obiettivo risiede nel replicare una realtà fisica, seppur circoscritta, 
attraverso criteri iconico-semantici e metrici. Lo scopo è teso a superare ciò che la vista non riesce a cogliere pienamente e che ne impedirebbe la riproduzione, la specificità della registrazione.

Il passaggio dalla mappa al modello 3D è un'operazione di trasmutazione che noi abbiamo svolto nel solco tracciato, per altri versi, da Walter Benjamin (1995), il quale ha sostenuto che tradurre è trasportare da un dominio linguistico a un altro un significato. Il modello digitale assume, quindi, la funzione di mediatore dei contenuti inafferrabili appartenenti alle mappe storiche, e mantiene con queste ultime un forte legame. Il fine ultimo è di ridurre la distanza tra documento originale e fruitore, ponendo quest'ultimo in una condizione più agevole di consultazione e comprensione.

Dobbiamo altresì tener presente che la suddetta traduzione permette anche di cogliere meglio altre analoghe situazioni (Russo, 2013).

$\mathrm{Ne}$ consegue una forma di sopravvivenza dell'immagine della Piazzaforte di Pescara, come per altre fortezze scomparse, una sorta di rivalutazione e di riconoscimento del portato militare ormai desueto, nonché uno stimolo all'esperienza cognitiva ed emotiva. (fig. 7)

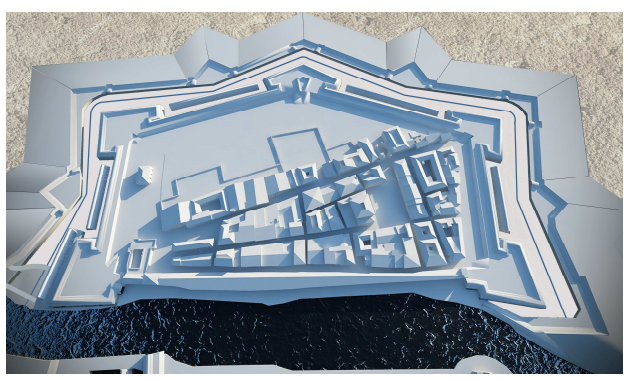

Fig. 7. Elaborazione 3D in itinere dell'area sud della Piazzaforte di Pescara (R. Potenza, P. Tunzi 2014-18).

Le fonti restano pertanto il riferimento unico e inequivocabile per poter svolgere qualunque attività di analisi, permettendo un'esegesi da cui potrà essere generata una nuova figurazione in cui il concetto di relazione tra le parti è conservato, nella forma e nel significato.

A giusta ragione Angela Maiello (2016) sottolinea la multi funzione dei nuovi media precisando che sono "dispositivi di distanziamento e riappropriazione, registrazione e rielaborazione di materiale mediale, visivo e audiovisivo, sistemi per lo sviluppo di nuove pratiche di elaborazione individuale e collettiva della memoria".

\section{Bibliography}

Benjamin, W. (1995). Il compito del traduttore, in Angelus Novus, Einaudi, Torino. p. 39

Giammusso, F.M. (2014). "La ricostruzione virtuale come strumento per l'analisi storica dell'architettura", INFO$L I O, 31$, p. 43.

Le Goff, J.; Seta, C. De. (1989). La città e le mura, Laterza, Roma-Bari, p. 1

Maiello, A. (2016). "L'immagine d'archivio nell'epoca della partecipazione interattiva", Rivista di Estetica, 63, in https://journals.openedition.org/estetica/1281\#bodyftn1 (05 August 2019).

Nietzsche, F. (1979). Sull'utilità e il danno della Storia per la vita, Adelphi, Milano, p. 11

Parisi, G. (1802). Elementi di Architettura militare del Maresciallo Giuseppe Parisi, D. Campo, Napoli.

Pessolano, M.R. (2006). Una fortezza scomparsa, Carsa edizioni, Pescara, p. 108

Potenza, R. (2015). "La fortezza di Pescara ricostruita", in Tunzi, P. ed., Pescara e il suo doppio, Carsa, Pescara, p. 45.

Rossi, P. (1991). Il passato, la memoria, l'oblio, Il Mulino, Bologna, p. 14

Russo, M. (2013). 'La rinascita dell'architettura Cham: un percorso di ricostruzione virtuale di architetture scomparse", Disegnare idee immagini, 46, p. 82. 